\title{
Microbial flora of the lower genital tract of women in labour in Zaria, Nigeria
}

\author{
CC EKWEMPU,* RV LAWANDE, $†$ AND LJ EGLER $\dagger$ \\ From *the Department of Obstetrics and Gynaecology, and the Department of Microbiology, \\ Ahmadu Bello University Hospital, Zaria, Nigeria
}

SUMMARY Nine genera of microbes isolated from the lower genital tract of 187 women in labour in Zaria have been identified. The work was undertaken to establish the nature of microorganisms in the lower genital tract of women in labour as a basis for further study. The isolates in order of prevalence were: Candida albicans (20.9\%), Klebsiella sp (15.0\%), Escherichia coli $(9 \cdot 1 \%)$, Streptococcus faecalis $(6.4 \%)$, haemolytic streptococci (other than Streptococcus pyogenes) $(2 \cdot 7 \%)$, Streptococcus viridans $(2 \cdot 1 \%)$, Staphylococcus aureus $(2 \cdot 1 \%)$, Aeromonas hydrophila $(2 \cdot 1 \%)$, Proteus mirabilis $(1 \cdot 1 \%)$, Peptostreptococcus putridus $(1 \cdot 1 \%)$, Streptococcus pyogenes $(0 \cdot 5 \%)$, and Streptococcus pneumoniae $(0.5 \%)$. Neisseria gonorrhoeae, Haemophilus $\mathrm{sp}$, Lactobacillus $\mathrm{sp}$, and Clostridium sp were sought but not found. Chlamydia, viruses, and T-strains of mycoplasma and trichomonas were not sought. It appears from this study that the lower genital tract of most women in Zaria at the time of labour is heavily colonised by pathogens. For this reason alone prolonged labour and trauma to the genital tract at the time of delivery should be avoided.

Puerperal sepsis is the single most common cause of illness and death among mothers in Zaria. Many of those affected have had no antenatal care and have had their labour supervised by traditional midwives. Often they come to hospital with severe genital infections or at the end of prolonged labour.

Many of these infections do not respond to antibiotics commonly used in the hospital, viz, penicillin, streptomycin, chloramphenicol, and occasionally ampicillin. One of the reasons for this lack of response may be the fact that the nature of the organisms concerned and their antimicrobial susceptibility are not known.

In order to ascertain the nature of these organisms a preliminary bacteriological investigation of the lower genital tract of women in labour in Zaria was undertaken.

Accepted for publication 9 July 1980

\section{Material and methods}

In a microbiological study of 187 women admitted in labour to Ahmadu Bello University teaching hospital in Zaria, cervical swabs were taken by circumferential sweep around the margin of the external os, placed in $5 \mathrm{ml}$ Stuart's transport medium, and sent to the laboratory.

Primary media were inoculated and incubated as shown in Table 1. All isolates were identified according to the methods recommended by Cowan and Steel. ${ }^{1}$

Chlamydia, viruses, and T-strain mycoplasmas and trichomonas were not sought in this study, and only simple methods of anaerobic culture were used.

\section{Results}

Nine groups or genera of microbes were isolated, as shown in Table 2. There were Candida albicans

Table 1 Media inoculated and incubated for primary cultures

\begin{tabular}{lllc}
\hline Medium & Atmosphere & Temperature $\left({ }^{\circ} \mathrm{C}\right)$ & Incubation time $(h)$ \\
\hline Blood agar & Aerobic, anaerobic, carbon dioxide & 37 & 24 to 48 \\
'Chocolate' agar & Aerobic, anaerobic, carbon dioxide & 37 & 24 to 48 \\
MacConkey's agar & Aerobic & 37 & 24 \\
Sabouraud's agar & Aerobic & 37 and 30 & 48 to 96 \\
\hline
\end{tabular}


Table 2 Organisms recovered from the lower genital tract of 187 women in labour

\begin{tabular}{lc}
\hline Organism & $\begin{array}{c}\text { No. (\%) of women with } \\
\text { positive cultures }\end{array}$ \\
\hline C. albicans & $39(20 \cdot 9)$ \\
Klebsiella sp & $28(15 \cdot 0)$ \\
E. coli & $17(9 \cdot 1)$ \\
Strep. faecalis & $12(6 \cdot 4)$ \\
Haemolytic streptococci (other than group A) & $5(2 \cdot 7)$ \\
Strep. viridans & $4(2 \cdot 1)$ \\
Staph. aureus & $4(2 \cdot 1)$ \\
Aeromonas hydrophila & $4(2 \cdot 1)$ \\
Proteus mirabilis & $2(1 \cdot 1)$ \\
Peptostreptococcus putridus & $2(1 \cdot 1)$ \\
Strep. pyogenes (group A) & $1(0 \cdot 5)$ \\
Strep. pneumoniae & $1(0 \cdot 5)$ \\
Number of women with negative cultures & $68(36 \cdot 4)$ \\
Number of women with positive cultures & $119(63 \cdot 6)$ \\
\hline
\end{tabular}

(21\%), Escherichia coli (9\%), Streptococcus faecalis $(6 \%)$, haemolytic streptococci (other than Streptococcus pyogenes) (3\%), Streptococcus viridans $(2 \%)$, Staphylococcus aureus $(2 \%)$, Aeromonas hydrophila ( $2 \%)$, Proteus mirabilis ( $2 \%)$, and Peptostreptococcus putridus ( $2 \%$ ), while Streptococcus pyogenes and Streptococcus pneumoniae constituted less than $1 \%$ of the isolates.

Neisseria gonorrhoeae, Haemophilus sp, Lactobacillus sp, and Clostridium sp were sought but not found. In all, $64 \%$ of the women yielded significant cultures.

\section{Discussion}

This work was undertaken in order to establish the microbial flora of the lower genital tract of women in labour in Zaria as a basis for further study.

Nine groups or genera of microorganisms were identified. The isolation of Klebsiella species and $E$. coli from so many women is rather surprising. According to Weinstein, ${ }^{2}$ de Louvois et al., ${ }^{3}$ and Goplerud et al., ${ }^{4}$ Klebsiella species and E. coli are not usual in the vagina. It is known that antibiotics may modify the vaginal flora, but most of the women in this study had had no antenatal care. They were admitted at the end of prolonged labour, and it is unlikely that they had received any antibiotics that might have affected the vaginal flora. The presence of many of the organisms found may reflect poor general hygiene.

The absence of lactobacilli from among the isolates in this study is also unusual, although it is known that lactobacilli are less frequently isolated elsewhere from women with disease of the lower genital tract. ${ }^{3}$ One explanation for this may be the $\mathrm{pH}$ of the vagina, which is known to become alkaline in certain morbid states. ${ }^{5}$

Isolation of Strep. faecalis, Strep. pyogenes, and Staph. aureus in this study is not surprising as these organisms are well-known aetiological agents of puerperal sepsis.
In recent years, there have been many reports on the possible rôle of Bacteroides species and related organisms in gynaecological and obstetric infections. $^{6-8}$ In the present study, Bacteroides species were not isolated by means of the routine anaerobic method used. Another anaerobic organism, Peptostreptococcus putridus, was isolated from the lower genital tract of two women. It seems surprising that colonisation of the lower genital tract with anaerobes does not give rise to major problems in this part of the world. Nevertheless, a more detailed investigation of the possible rôle of anaerobic organisms in genital tract infections in this geographical locality is desirable.

High recovery of $C$. albicans in this study conforms with the findings of other workers, ${ }^{39}$ and the association of $C$. albicans with disease of the lower genital tract in pregnancy is well known.

Recovery of Aeromonas hydrophila from the lower genital tract of four women is interesting. This organism is a well-documented human pathogen causing wound infections and septicaemia, but its association with genital tract infections is not very common.

Perhaps the most important fact that has emerged from this work is that the lower genital tract of most women in Zaria at the time of labour is heavily colonised by pathogens. In order to prevent genital tract sepsis, therefore, labour should not be prolonged, and unnecessary trauma to the genital tract at the time of delivery should be avoided.

\section{References}

1 Cowan ST, Steel KJ. Manual for the Identification of Medical Bacteria. London: Cambridge University Press, 1974.

2 Weinstein L. The bacterial flora of the human vagina. Yale J Biol Med 1938;10:247.

${ }^{3}$ de Louvois J, Hurley R, Stanley VC. Microbial flora of the lower genital tract during pregnancy: relationship to morbidity. J Clin Pathol 1975;28:731-5.

${ }^{4}$ Goplerud CP, Ohm MJ, Galask RP. Aerobic and anaerobic flora of the cervix during pregnancy and puerperium. Am J Obstet Gynaecol 1976;126:858-68.

${ }^{5} \mathrm{Karnaky} \mathrm{KJ}$. Electronic studies in obstetrics and gynaecology. Obstet Gynaecol 1955;5:93-101.

- Neary MP, Allen J, Okubadejo OA, Payne DJH. Preoperative vaginal bacteria and post operative infections in gynaecological patients. Lancet $1973 ; 2: 1291-4$.

7 Thadepalli H, Gorbach SL, Keith L. Anaerobic infections of the female genital tract: bacteriologic and therapeutic aspects. Am J Obstet Gynaecol 1973;117:1034-40.

${ }^{8}$ Craft I, Ghandi F, Hardy R. Bacteroides in gynaecological infections. Lancet 1974;1:677.

- Stanley VC, Hurley R, Carroll CJ. Distribution and significance of Candida precipitins in sera from pregnant women. J Med Microbiol 1972;5:313-20.

Requests for reprints to: Dr CC Ekwempu, Department of Obstetrics and Gynaecology, Ahmadu Bello University, PMB 1206, Zaria, Nigeria. 